PROCEEDINGS OF THE

AMERICAN MATHEMATICAL SOCIETY

Volume 137, Number 12, December 2009, Pages 4259-4273

S 0002-9939(09)09960-2

Article electronically published on July 15, 2009

\title{
ON SPUN-NORMAL AND TWISTED SQUARES SURFACES
}

\author{
HENRY SEGERMAN
}

(Communicated by Daniel Ruberman)

\begin{abstract}
Given a 3 manifold $M$ with torus boundary and an ideal triangulation, Yoshida and Tillmann give different methods to construct surfaces embedded in $M$ from ideal points of the deformation variety. Yoshida builds a surface from twisted squares, whereas Tillmann produces a spun-normal surface. We investigate the relation between the generated surfaces and extend a result of Tillmann's (that if the ideal point of the deformation variety corresponds to an ideal point of the character variety, then the generated spunnormal surface is detected by the character variety) to the generated twisted squares surfaces.
\end{abstract}

\section{INTRODUCTION}

Yoshida in [6] and Tillmann in 3 describe different methods of producing surfaces within a 3-manifold $M$ with boundary from ideal points of the deformation variety of $M$, given a particular ideal tetrahedralisation of $M$. The Yoshida construction builds a surface from twisted squares within the tetrahedra, and the Tillmann construction results in a spun-normal surface relative to the tetrahedra. In this paper we investigate the connection between the methods. We show first that a twisted squares surface can be obtained from the spun-normal surface by an isotopy and possibly two different types of compression moves (the usual disk compression moves, and compressions along certain annuli):

Theorem 1.1. For a 3-manifold $M$ with $\partial M$ a union of tori, if $T$ is a twisted squares surface and $S$ the spun-normal surface produced from the same data, then $T$ can be obtained from $S$ by the correct choices made of isotopy and a finite number of compressions along disks and boundary annuli, and removing boundary parallel components.

Both constructions, with a mild condition on the ideal point we start from, result in surfaces which although they may not be essential, can be reduced to essential surfaces by (disk) compressions and possibly deleting components 11 Tillmann shows that if the ideal point of the deformation variety corresponds to an ideal point of the

Received by the editors October 10, 2008, and, in revised form, March 7, 2009.

2000 Mathematics Subject Classification. Primary 57M99.

The author was partially supported by an NSF-RTG postdoctoral fellowship.

1 There are two different senses of an "essential" surface in the literature which differ in whether or not they require the surfaces to be boundary incompressible or not. Yoshida does require boundary incompressibility (and refers to the surfaces as "incompressible and boundary incompressible"), whereas Tillmann does not. We will use the version that does not require boundary incompressibility in this paper, although all of the arguments also go with the other version. 
character variety, then the corresponding spun-normal surface is dual to the ideal point of the character variety in the sense of Shalen [2. We use Tillmann's result to show that the same is true for twisted squares surfaces produced by Yoshida's construction, and so any essential surface obtained from either the spun-normal or twisted squares construction in this case is also detected by the character variety:

Theorem 1.2. Let $M$ be an oriented 3-manifold with $\partial M$ a union of tori with ideal triangulation $\mathcal{T}$ and $T$ a two-sided twisted squares surface obtained via Yoshida's construction from an ideal point of the deformation variety $\mathfrak{D}(M, \mathcal{T})$ which corresponds to an ideal point of the character variety. Then any essential surface obtained from $T$ by compressions is detected by the character variety.

See section 5 for details. We use this in [1] to show that all non-fiber and nonsemi-fiber incompressible surfaces in punctured torus bundles are detected by the character variety. The author thanks Cameron Gordon and Stephan Tillmann for helpful discussions.

1.1. The projective admissible solution space and spun-normal form. Spun-normal surfaces are made up of two types of surface parts which sit within the tetrahedra of the ideal tetrahedralisation: triangles and quadrilaterals. The data that Tillmann uses to produce a spun-normal surface is the number and positioning of quadrilaterals in each tetrahedron, subject to the "Q-matching equations" (see Tillmann [4). The projective admissible solution space is the set of solutions to the Q-matching equations such that at most only one type (positioning) of quadrilateral appears in each tetrahedron (this makes the solution "admissible") and normalised so that the sum of the coordinates is one. Given such a solution with rational coordinate ratios, the solution is scaled again so that the coordinates are integers, with greatest common divisor of one, and these give us the number and type of quadrilaterals to use.

Theorem 2.4 of [4] states that an admissible integer solution of the Q-matching equation $\$ 2$ corresponds to a spun-normal surface. The surface is constructed by inserting the appropriate number of quadrilateral pieces and infinitely many triangle pieces in each tetrahedron, making sure that the boundaries of those pieces can be made to match up by isotoping them within each tetrahedron, and then removing any boundary parallel components.

1.2. The Yoshida construction. The Yoshida construction in [6] uses the same data (an admissible integer solution to the Q-matching equations, although Yoshida does not use this terminology) but in a different way to produce what we call a twisted squares surface. In place of a number of parallel quadrilaterals within a given tetrahedron, we put the same number of parallel twisted squares. See Figure 1.

The slopes of corners of quadrilaterals incident at an edge go in either a right hand or left hand screw direction around the edge, and the Q-matching equations for spun-normal surfaces state that the numbers of these incident at an edge are equal (counting quadrilaterals from all tetrahedra incident at that edge). If we push each edge of the quadrilateral towards the single vertex of the face of the tetrahedron cut off by that edge (this is the idea of the first isotopy; see section 2.11), then the quadrilateral becomes a twisted square, each right hand screw direction slope

${ }^{2}$ This is the "data" referenced in Theorem 1.1 

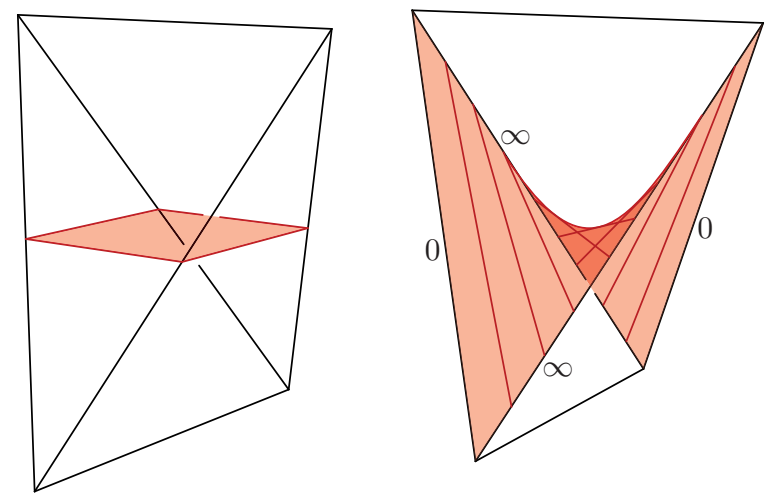

Figure 1. A tetrahedron with a quadrilateral and a tetrahedron with the corresponding twisted square, 0 and $\infty$ edges labelled.

becomes a 0-edge of a twisted square, and each left hand screw direction slope becomes an $\infty$-edge.

The Q-matching equations ensure that the pieces of a spun-normal surface match up around an edge of the tetrahedralisation. These equations correspond to the requirement in the Yoshida construction that the number of 0 -edges matches the number of $\infty$-edges of twisted squares that meet at each edge of the tetrahedralisation 3

Having placed the appropriate twisted squares within the tetrahedra, the Yoshida construction continues by removing parts of the twisted squares within a small tubular neighbourhood $\mathcal{N}_{e}$ of each edge $e$, then joining the resulting edges of the twisted squares to each other using long thin strips parallel to $e$ which each link a 0 -edge to an $\infty$-edge. See Figure 2 for an example which shows that there may be choices in this step of the construction.
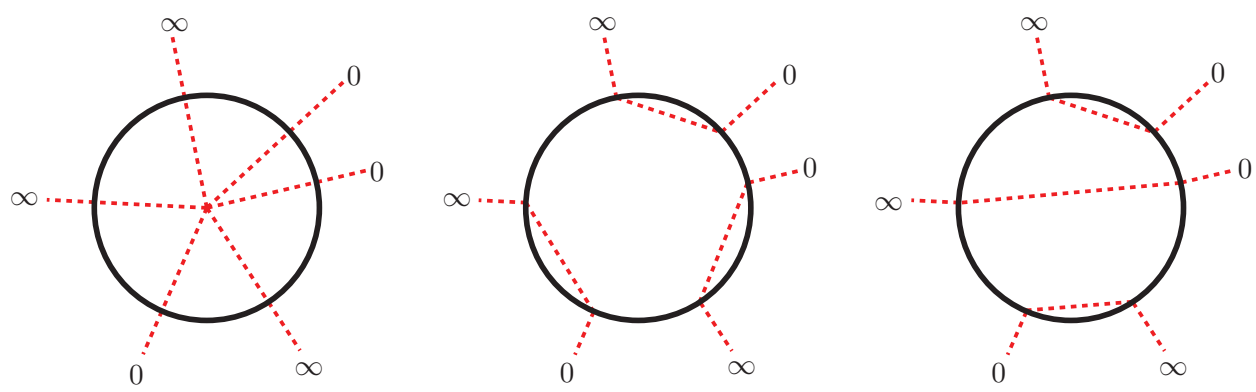

Figure 2. The cross-section view of parts of twisted squares within an $\mathcal{N}_{e}$ before removing them, and two different ways to rejoin 0 and $\infty$ edges of twisted squares to each other with long thin strips.

\footnotetext{
${ }^{3}$ Yoshida labels the edges of each twisted square this way in reference to the limits of complex dihedral angles of the tetrahedra as we approach an ideal point of the deformation variety. This matching condition at an edge corresponds to the fact that the product of complex dihedral angles of tetrahedra around the edge remains bounded (in fact equal to 1) as we approach the ideal point.
} 
The last step of the construction deals with what happens near the cusp(s). First, we truncate all of our tetrahedra, which also cuts off the corners of the twisted squares. The resulting triangulation of the boundary of the manifold contains boundaries of the twisted squares and boundaries of the long thin strips, which together form curves. We look for any null-homotopic curves here, and starting with the innermost such curve, we cap each off with a disk and push the disk inside the manifold. This completes the Yoshida construction of a twisted squares surface.

Remark 1.3. Both Tillmann and Yoshida in their papers generating essential surfaces deal with any one-sided surfaces they generate by taking double covers, Tillmann by scaling the admissible rational solution to the Q-matching equations by the minimal integer to result in a two-sided surface, and Yoshida by taking a double cover of any one-sided components of the twisted squares surface generated. We do not assume these steps in the construction of spun-normal or twisted squares surfaces. They also both go on to perform compressions (and boundary compressions for Yoshida) in order to produce incompressible surfaces, and again we do not take these steps.

\section{THe ISOTOPY}

We begin with a surface $S$ in spun-normal form relative to an ideal tetrahedralisation $\mathcal{T}$ of a 3 -manifold $M$ with boundary $\partial M$. We consider the tetrahedra as truncated but with "open ends" so that the infinitely many triangle pieces of the spun-normal surface will not accumulate within the manifold. See Figure 3 , Let $\mathcal{E}$ be the edge set of $\mathcal{T}$ and let $\mathcal{N}_{e}$ be small disjoint tubular neighbourhoods of the edges $e \in \mathcal{E}$. For each $f$ in the edge set of the spun-normal surface, let $\mathfrak{N}_{f} \subset S$ be a small 2-dimensional neighbourhood of $f \backslash\left(\mathcal{N}_{e} \cup \mathcal{N}_{e^{\prime}}\right)$ where $e, e^{\prime} \in E$ are the two edges of $\mathcal{T}$ at either end of $f$. We choose these neighbourhoods so that for $f \neq f^{\prime}, \mathfrak{N}_{f}$ and $\mathfrak{N}_{f^{\prime}}$ are disjoint. The triangles of $S$ are thus divided into parts within an $\mathcal{N}_{e}$, parts within an $\mathfrak{N}_{f}$, and a central region, which for a triangle $t$ is denoted $\mathfrak{C}_{t}$. The quadrilaterals are similarly divided.

We will describe an isotopy of $S$ which will convert all of the quadrilaterals in $S$ into twisted squares. The triangles of $S$ will correspond to either parts of disks which cap off null-homotopic boundary curves of the resulting twisted squares or parts of the surface which spiral around into the cusp.

2.1. First stage of the isotopy. See Figure 4. In the left diagram we have four tetrahedra arranged around a central vertical edge and part of a spun-normal surface consisting of two quadrilaterals and two triangles, with one of the triangles and one of the quadrilaterals shaded. In the right diagram we have the first stage of the isotopy, with the tetrahedra removed for clarity. The two triangles have been pushed towards the top vertex, the two quads have become the saddle shapes, with one shaded and one not. We describe in general how to perform this first step as follows:

First, fix the parts of the surface inside all the $\mathcal{N}_{e}$ (the components are all parallel disks that cut across the tube). Each edge $f$ in the edge set of the spun-normal surface $S$ is a normal curve in a face of $\mathcal{T}$, and so there is one vertex of that face between the two ends of $f$. We pull the $\mathfrak{N}_{f}$ towards that vertex. At the same time we extend the isotopy to pull any $\mathfrak{C}_{t}$ towards the vertex they are nearest to, together with any $\mathfrak{N}_{f^{\prime}}$ for edges $f^{\prime}$ parallel to $f$ on parallel sheets of $S$. We also 


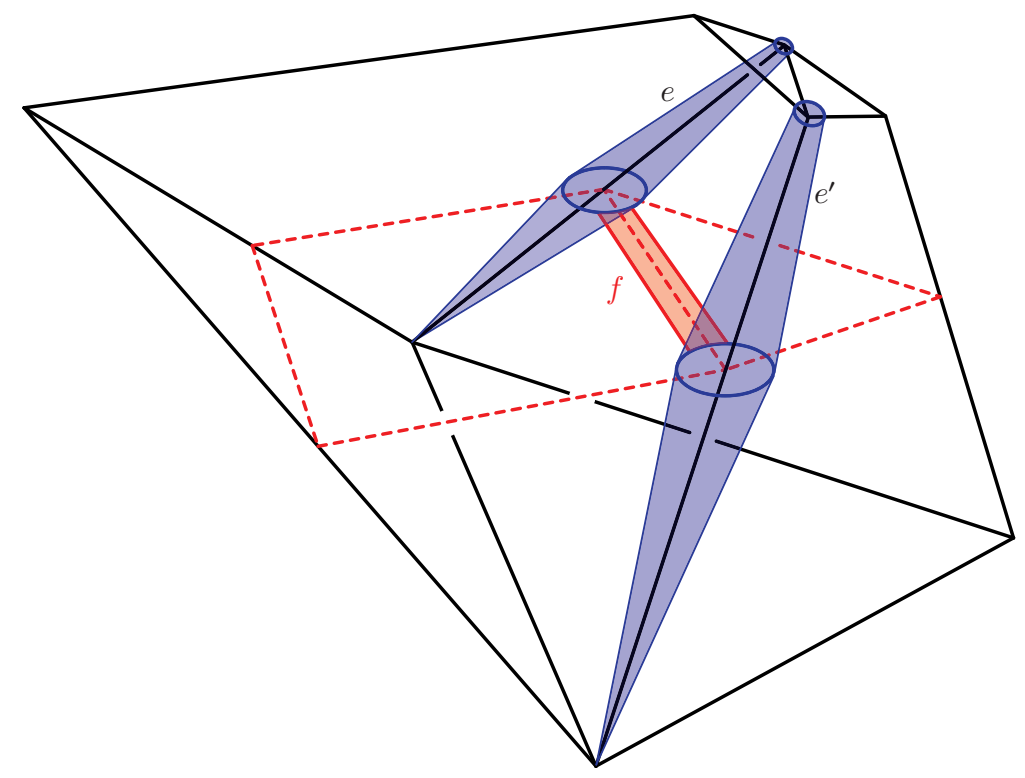

Figure 3. Two tetrahedra with a quadrilateral and triangle. Labelled are two edges of $\mathcal{T}, e$ and $e^{\prime} . \mathcal{N}_{e}$ and $\mathcal{N}_{e^{\prime}}$ are shaded. Also labelled is the edge between the quadrilateral and the triangle, $f$, and $\mathfrak{N}_{f}$ is shaded.
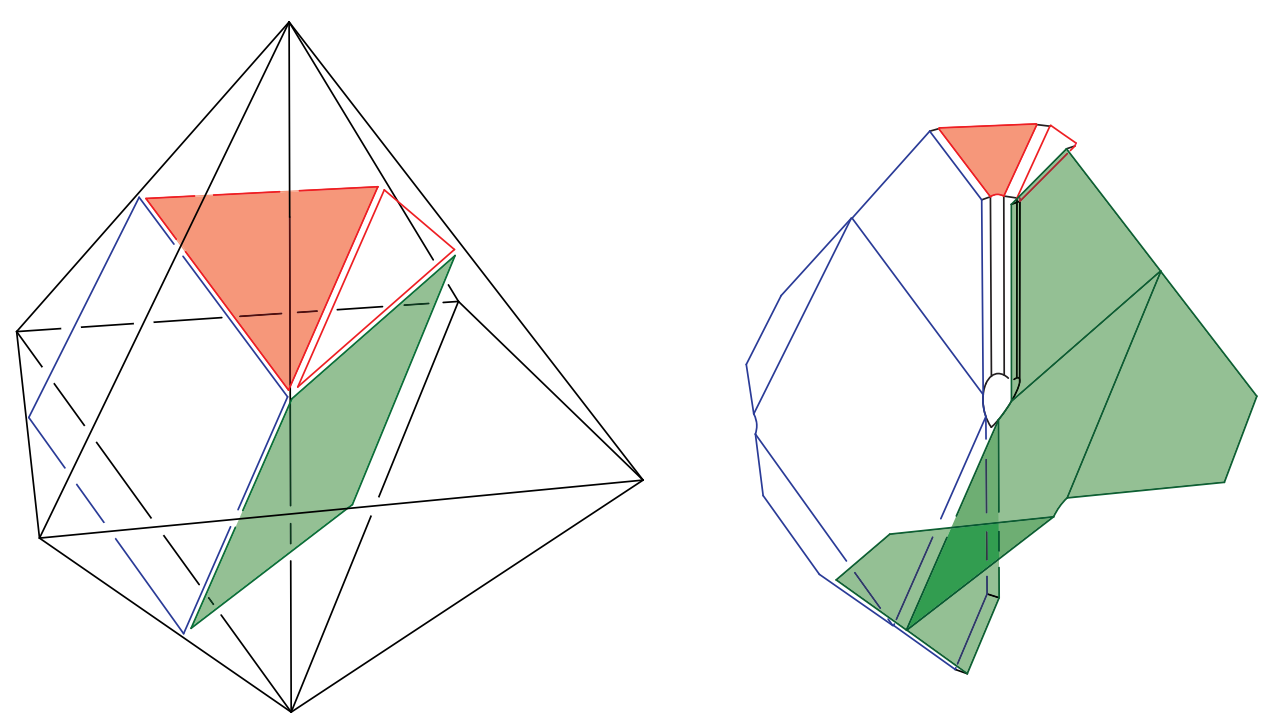

Figure 4. Part of a surface in spun-normal form and the first stage of the isotopy.

pull all parallel $\mathfrak{C}_{t^{\prime}}$ towards their vertex, again leaving fixed the parts of $S$ within the $\mathcal{N}_{e}$. We continue to refer to pieces of surface in the isotoped $S$ as $\mathfrak{N}_{f}$ and $\mathfrak{C}_{t}$. 
We cannot of course break the surface as we pull parts of it towards the vertices, so as we pull we introduce strips in a small neighbourhood of the boundary between the $\mathfrak{N}_{f}$ and $\mathfrak{C}_{t}$ and the rest of the surface (the parts inside the $\mathcal{N}_{e}$ and the rest of the quadrilaterals) in order to maintain the connection between these parts. The quadrilaterals together with the added strips become twisted squares, and we obtain the right hand diagram of Figure 4

2.2. Second stage of the isotopy. In the second stage we need to deal with the surface parts inside the $\mathcal{N}_{e}$ in order for us to join the twisted squares we get from the first stage to each other using long thin strips parallel to the edges $e$.

In the example of Figure 4 we obtain two twisted squares that meet at the vertical edge. In the Yoshida construction, these are to be joined by a long thin strip of surface, and in this case there is only one way to do it. Here the parts inside $\mathcal{N}_{e}$ and strips are isotopic (this is the second stage of the isotopy) to a single long thin strip parallel to the vertical edge and joining the two twisted squares.
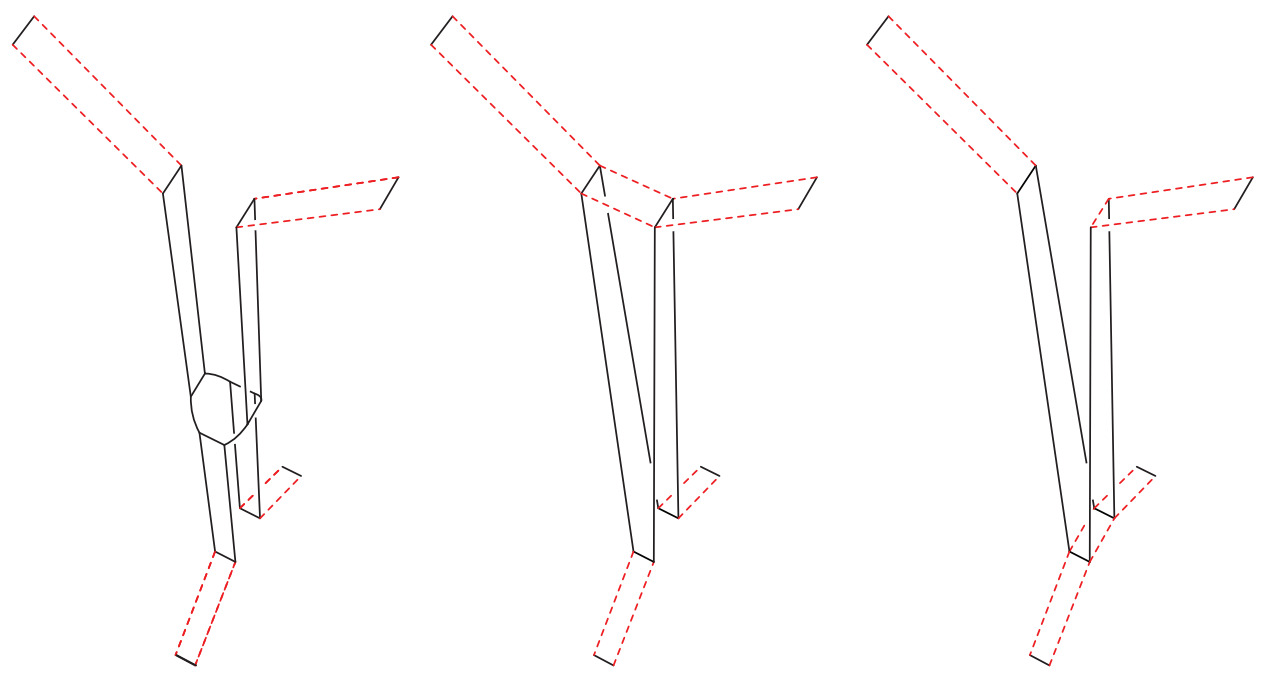

Figure 5. Four quadrilateral pieces after the first stage of the isotopy; only the $\mathfrak{N}_{f}$ and the disk within $\mathcal{N}_{e}$ are shown. These are the two ways to convert this into long thin strips parallel to $e$.

A more complicated example is shown in Figure 5. Here four quadrilaterals meet at the vertical edge, and the left hand diagram shows the picture after the first isotopy. Figure 6 shows the twisted squares we get. Here, we get a choice as to how to deal with the disk within the $\mathcal{N}_{e}$ in the second part of the isotopy: we can either push it up, as in the center diagram, or down, as in the right hand diagram. These correspond to the two ways in which to join the four edges of the twisted squares with long thin strips parallel to the vertical edge.

In general we will have some number of parallel disks in each $\mathcal{N}_{e}$, each with strips attached to the edge making the disk into a saddle of some order. The second stage of the isotopy consists of pushing the various disks to one end of the edge or the other (in fact disks can be split apart into two or more regions, each of which can go to either end of the edge). We will investigate this in the next section. 


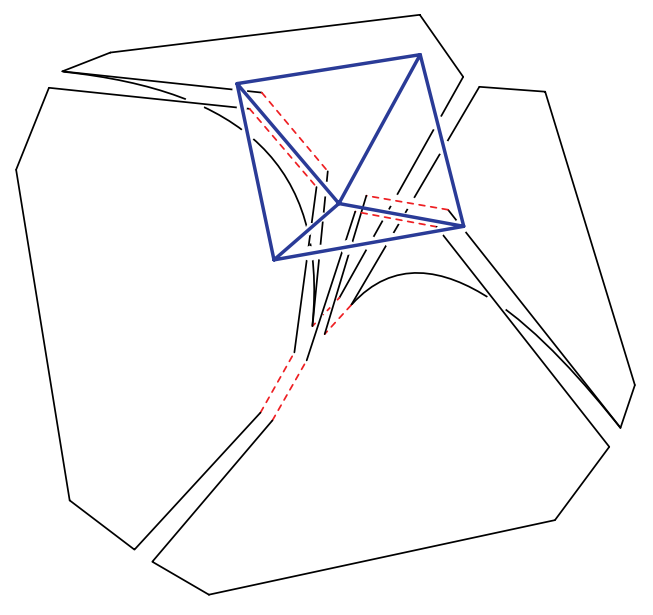

Figure 6. Four twisted squares. The truncated ends of the tetrahedra at the top vertex are shown.

\section{Choices in the Yoshida construction}

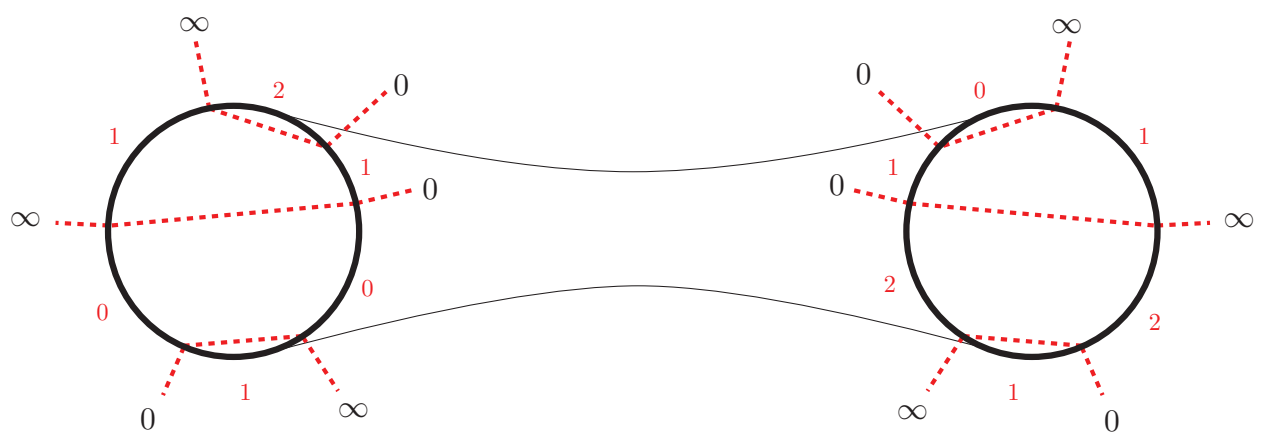

Figure 7. Two ends of a $\mathcal{N}_{e}$ with a choice of long thin strips.

The choices to be made in constructing a given Yoshida form surface are which edges of twisted squares to join with each other. Given a choice of those joins we will decide how to push each disk of the surface after the first stage of the isotopy in order to realise those joins. Consider Figure 7, which shows both ends of an $\mathcal{N}_{e}$ with a choice of long thin strips. Note of course that the picture at one end is a mirror reflection of the picture at the other end. We have added integers around each end, with an integer in between each sheet of the twisted squares meeting at $e$. Moving anti-clockwise around the end, the integer increases by one when we pass a 0 incoming edge and decreases by one for each $\infty$ edge. Because the number of 0 edges is equal to the number of $\infty$ edges, these integers can be chosen consistently; and if we require that the smallest integer be 0 , then they are uniquely defined.

If the maximum number we reach is $n$, then the corresponding number at the other end of $e$ to an integer $k$ is $n-k$. Notice that $n$ is also the number of disks that sit in the center of $e$ after the first step of the isotopy from spun-normal form. 
Observe also that the disk at an end of $\mathcal{N}_{e}$ is cut into a number of regions by the ends of the long thin strips and the integers on the boundary of a region are all the same, so we associate that integer with the whole region. This information tells us how to push the disks from the center of $e$ out to the ends: we deal with each region of the disks independently. For a region of the end with associated integer $k$, we push $k$ sheets to this end and $n-k$ sheets to the other. The above observations show that we can consistently do this, and we end up with the desired long thin strips.

We now find ourselves at the end of the second isotopy from spun-normal form: we have isotoped so that all quadrilaterals are now twisted squares, joined to each other through the desired choice of long thin strips.

Theorem 3.1. Given a twisted squares surface $T$ and the spun-normal surface $S$ produced from the same data, $T$ and $S$ may be isotoped so that outside of a small neighbourhood of $\partial M$ in $M$ the two surfaces coincide.

Proof. The above isotopy of $S$ produces a surface $S^{\prime}$. Now delete from $S^{\prime}$ all $\mathfrak{N}_{f}, \mathfrak{C}_{t}$ and parts of the disks within the $\mathcal{N}_{e}$. We obtain a surface $S^{\prime \prime}$ which has boundary in the interior of the manifold $M$, but whose boundary is all close to $\partial M$. Choose a suitable product neighbourhood $N_{S}$ of $\partial M$ that contains the boundary of $S^{\prime \prime}$. Choose a suitable product neighbourhood $N_{T}$ of $\partial M$ which contains any capped off boundary curves of $T$. Then $S^{\prime \prime} \backslash\left(N_{S} \cup N_{T}\right)$ is combinatorially identical and hence isotopic to $T \backslash\left(N_{S} \cup N_{T}\right)$.

\section{Compressions}

We now need to understand the parts of the surface near the boundary, which is made out of the strips $\mathfrak{N}_{f}$, the central regions of triangles $\mathfrak{C}_{t}$ and parts of disks within the $\mathcal{N}_{e}$. For simplicity we restrict ourselves from now on to manifolds $M$ such that $\partial M$ is a union of tori. We consider the curves $C$ forming the boundary of $S^{\prime \prime}$ from the proof of Theorem 3.1. Let $N \cong \partial M \times[0,1)$ be a closed product neighbourhood of $\partial M$ such that $N_{0}:=\partial M \times\{0\} \subset N$ and $C \subset N_{0}$. We may put $S^{\prime}$ in the same picture in such a way that all $\mathfrak{N}_{f}, \mathfrak{C}_{t}$ and parts of the disks within the $\mathcal{N}_{e}$ are contained in $N$, their intersection with $N_{0}$ consists only of $C$ and the intersection of the rest of $S^{\prime}$ (twisted squares and long thin strips) with $N$ also consists only of $C$. Note that the surface within $N$ has a well defined side that faces the cusp and a side that faces into the manifold: no part of the surface is ever "vertical". The key observation is that the pattern of the curves $C$ against the triangulation of $N_{0}$ induced by $\mathcal{T}$ determines how and if those curves are connected together within $N$ by the $\mathfrak{N}_{f}$, the $\mathfrak{C}_{t}$ and parts of disks within the $\mathcal{N}_{e}$, as we will see in the following discussion.

In Figure 8 we see an example of part of the triangulation on $N_{0}$. The curves $C$ consist of the boundaries of twisted squares (which were once quadrilateral pieces), shown as dotted arcs in the triangular end of the tetrahedron containing the twisted square and alternating with the ends of long thin strips within $\mathcal{N}_{e}$ which are only shown for the central $e$. The strips $\mathfrak{N}_{f}$ join onto these arcs crossing over the edges of the triangulation. At the other side of the $\mathfrak{N}_{f}$ can be either another arc boundary of a twisted square (as on the left of the figure) or a $\mathfrak{C}_{t}$ (as on the right). Also in Figure 8 we have oriented the arcs in such a way that the arrow points anti-clockwise around the triangle of the triangulation of $N_{0}$ containing the arc (this orientation 


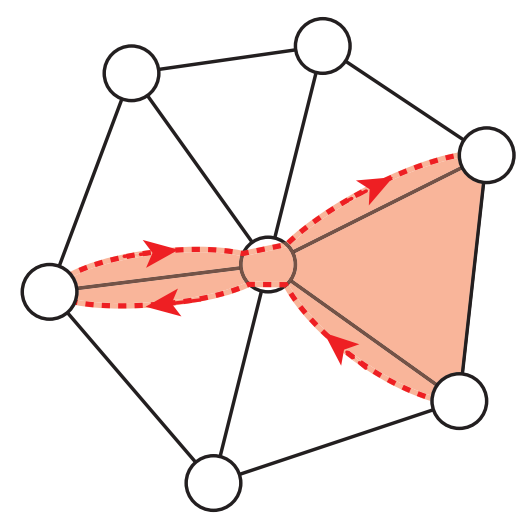

Figure 8. Parts of $C$ on $N_{0}$ as seen from the cusp looking inwards.

points from the edge of the twisted square labelled $\infty$ to the one labelled 0 ). Note that the orientation here is the same as the orientation of the boundary of the twisted squares on $\partial M$ in Yoshida [6] and that when we link the arcs at the $\mathcal{N}_{e}$ the orientations on the linked arcs agree. Beware however that this orientation is not induced by an orientation of the surface.

Notice that with this orientation the $\mathfrak{N}_{f}, \mathfrak{C}_{t}$ and parts of disks in $\mathcal{N}_{e}$ always meet the arc coming from the right of the arrows, and as mentioned before, the surface within $N$ is never "vertical", so the orientation of the surface within $N$ is consistent. The curves $C$ with this orientation thus determine the surface within $N$ : We can reconstruct the surface from $C$ by putting infinitely many layered copies of $N_{0}$ minus a small neighbourhood of $C$ into $N$ and connecting neighbouring layers to each other with annuli in a way determined by the orientation on each curve as in Figure 9. Figure 10 shows the same surface after an isotopy that simplifies the picture.

Definition 4.1. We denote by $\mathcal{S}(C)$ the surface embedded in $N$ determined by the oriented boundary curves $C$ as in the above discussion.

Definition 4.2. A boundary annulus for a surface $F$ in a 3-manifold $M$ with nonempty boundary $\partial M$ is an annulus $A$ embedded in $M$ with boundary components $\alpha \subset F$ and $\beta \subset \partial M$ and such that $A \cap F=\alpha$ and $A \cap \partial M=\beta$.

We also define a compression along a boundary annulus analogously to compression along a disk. That is, we cut the surface along $\alpha$, where it intersects the annulus and stitch in two parallel copies of the annulus. This move introduces two boundary components to the surface. See Figure 11.

Proof of Theorem 1.1. We start by isotoping $S$ and orienting the curves $C$ as in the discussion at the beginning of this section (the isotopy depends of course on the twisted squares surface $T$ that we are aiming for). Each torus boundary component of $M$ has its own component of $N$, and each curve on each component of $N_{0}$ falls into one of 3 categories:

(1) Null-homotopic curves with arrows pointing in the clockwise direction.

(2) Null-homotopic curves with arrows pointing in the anti-clockwise direction.

(3) Some number of parallel non-null-homotopic curves. 


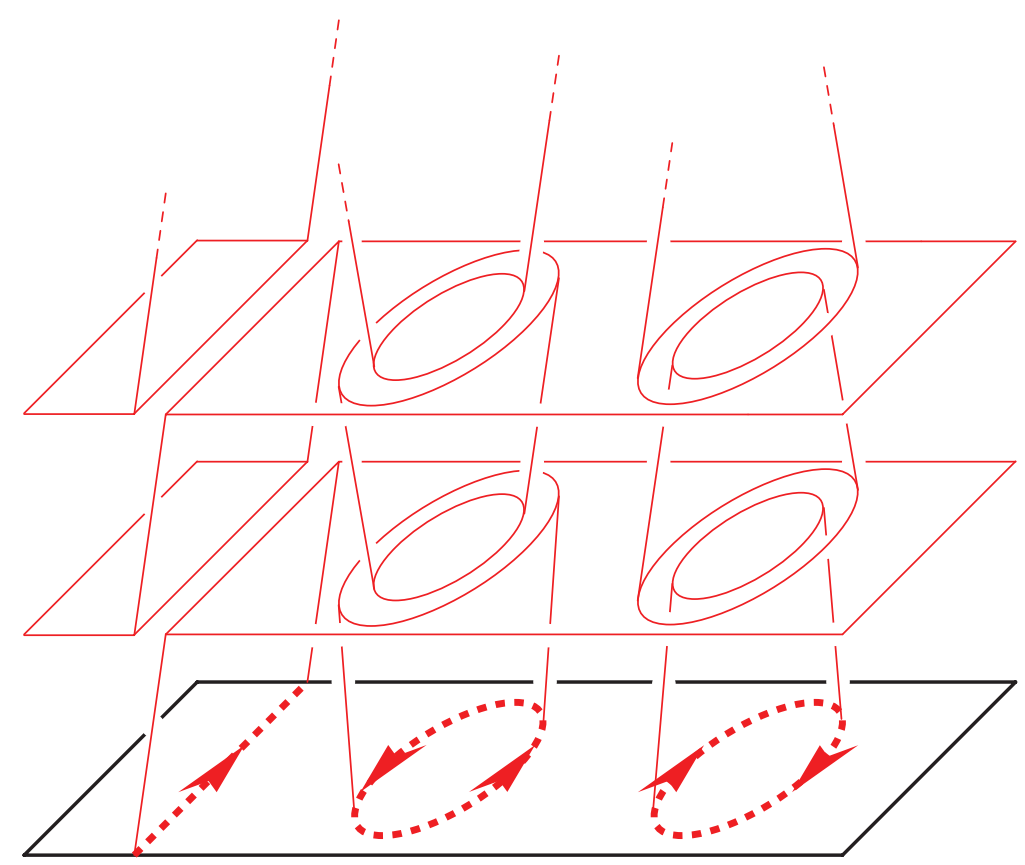

Figure 9 . The surface $\mathcal{S}(C)$ in $N$ determined by a given set of oriented curves $C \subset N_{0}$.

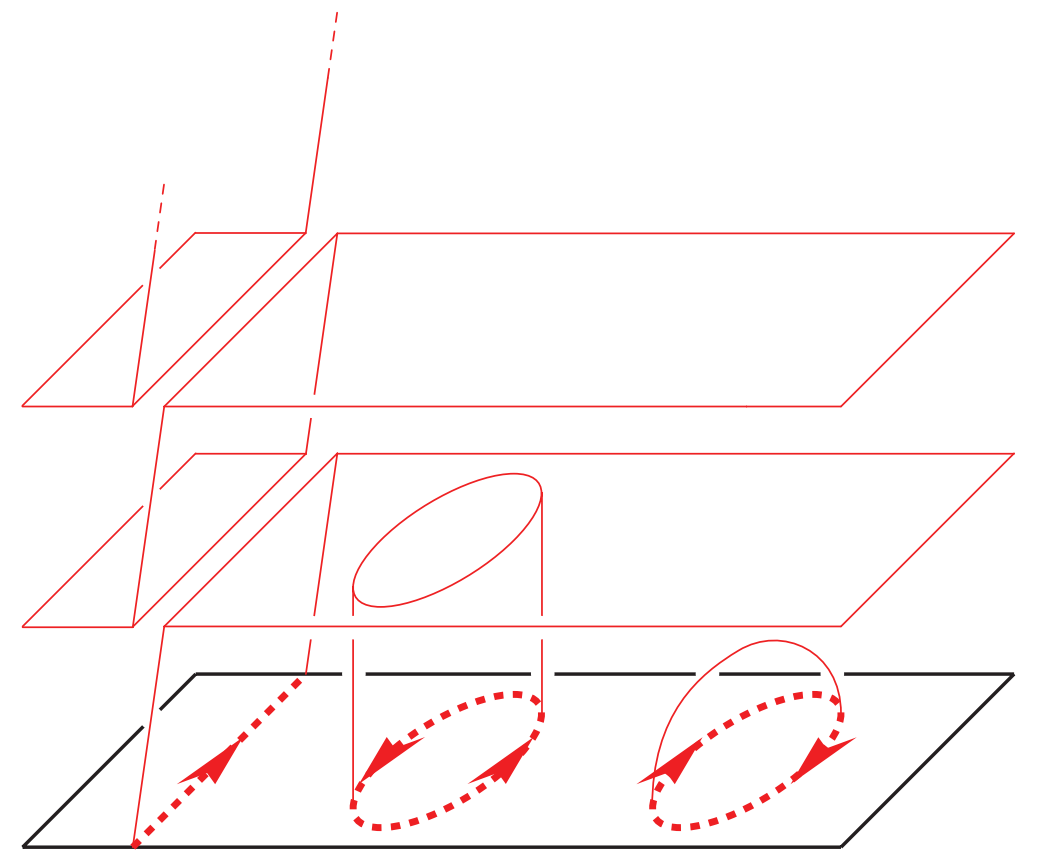

Figure 10. An isotope of the surface from Figure 9 . 

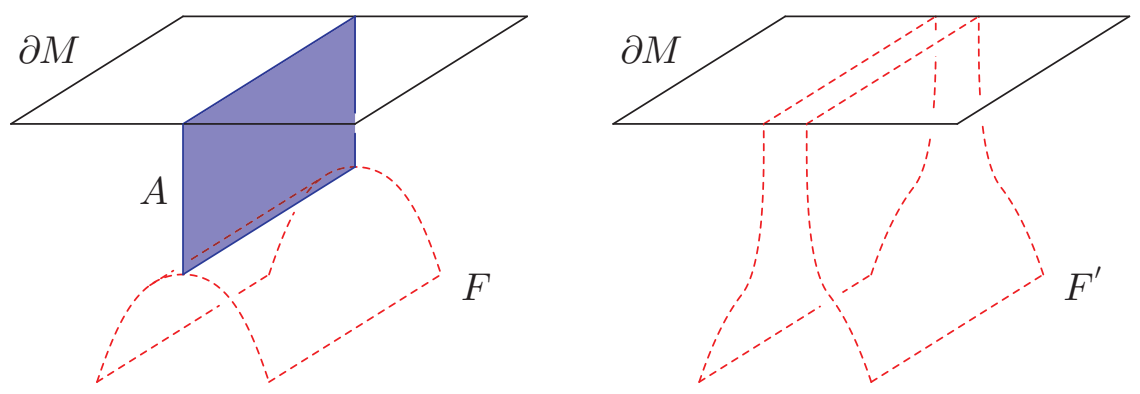

Figure 11. A boundary annulus $A$ (shaded) and the result of compression along it.

"Clockwise" and "anti-clockwise" are as seen from $\partial M$ looking down on $N_{0}$. The surfaces $S$ and $T$ are identical outside of $N$, but may differ within. We describe moves to change $S \cap N$ into $T \cap N$.

Given a single connected curve $C_{i}$ as in case (1) with no smaller curve contained within it, the component of $\mathcal{S}(C)$ connected to it caps off the curve in the same way as in the twisted square construction, and so no compression move needs to be made. See the right hand null-homotopic curve in Figure 10.

Given a single connected curve $C_{i}$ as in case (2) with no smaller curve contained within it, the component of $\mathcal{S}(C)$ connected to it goes outwards from the curve rather than inwards. We can perform a compression move on the disk bounded by this curve which caps off the curve on the inside, as in $T$. It also caps off the "outside" surface, removing a funnel going down to $N_{0}$. See the left hand null-homotopic curve in Figure 10

In the first case above, we may delete $C_{i}$ from $C$ without affecting the parts of the surface other than the cap bounded by the curve, so $\mathcal{S}\left(C \backslash C_{i}\right)$ is the same as the result of removing the cap from $\mathcal{S}(C)$. In the second case above we can do the same, in the sense that $\mathcal{S}\left(C \backslash C_{i}\right)$ is the same as the result of performing the compression move and then removing the cap from $\mathcal{S}(C)$. We perform these two moves to all the null-homotopic curves, innermost first.

We are left with only the curves of case (3) to deal with, the parts of the surface connected to the null-homotopic curves now matching with the parts of $T$ connected to those curves. The parts of the surface connected to the non-null-homotopic curves are at this point the same as if there were no null-homotopic curves at all, so the situation is as in the example of Figure12 We can pair up curves with opposite orientation that are connected to each other with annuli, leaving some number of curves without a partner, all of which must have the same orientation. The surface components attached to these curves are unbounded and spin infinitely many times around the torus boundary towards the cusp.

For the unbounded surface case we "unwind" the spinning with an isotopy which leaves the surface in the same state as if we had simply deleted the surface attached to the curve and replaced it with an annulus connecting the curve on $N_{0}$ straight out to $\partial M$. If there are no unbounded parts of the surface spinning out to the cusp, then there may be boundary parallel tori components of the surface, which we delete at this stage. 


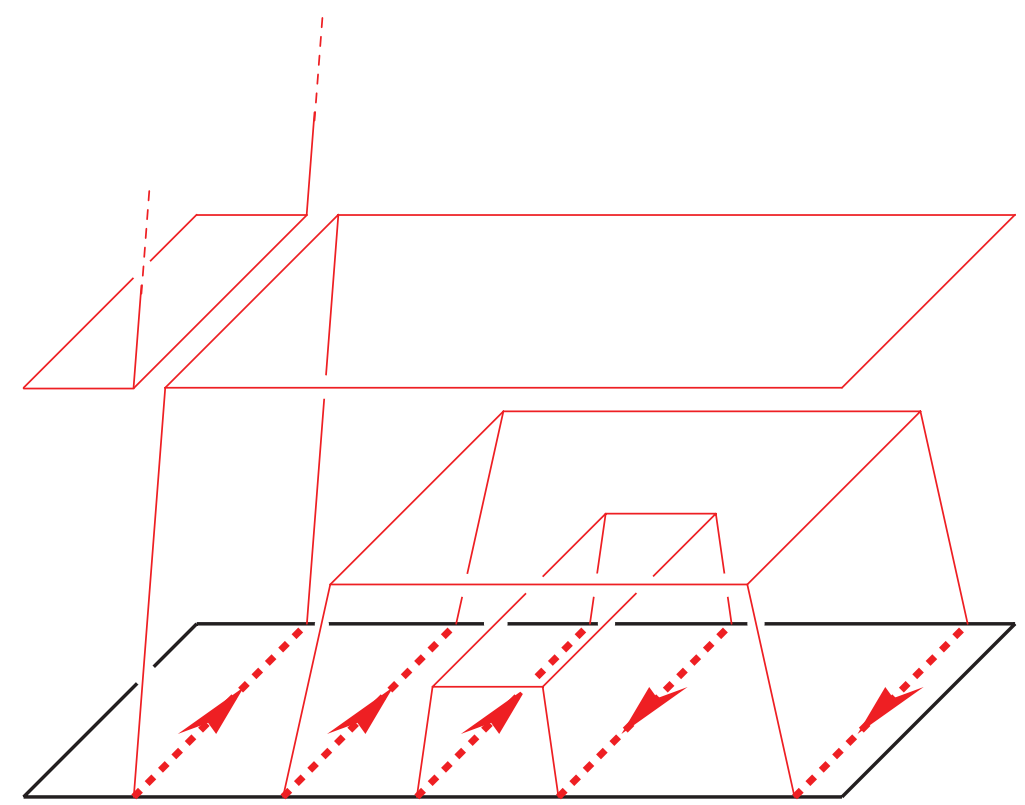

Figure 12. Curves in case (3) and the surface associated with them.

Finally, for the annular components of the surface in $N$, in order to recover $T$ we need to cut along the core of the annulus and move the resulting edges out to $\partial M$ (doing this for outermost annular components first). We do this by performing compressions along boundary annuli with one edge on the core of the annulus we are cutting and the other on $\partial M$, as in Figure 11. The resulting surface is $T$.

\section{ACtions on trees}

For this section, we use the phrase " $F$ is dual to an action of $\pi_{1} M$ on a tree T" in the same sense as Shalen [2], section 2.2. That is, we can construct the surface $F$ in the following way: We have a tree $\mathfrak{T}$, with $E$ the set of midpoints of edges of $\mathfrak{T}$ and a map $\widetilde{f}: \widetilde{M} \rightarrow \mathfrak{T}$ which is $\pi_{1} M$ equivariant and transverse to $E$, and $\widetilde{f}^{-1}(E)=p^{-1}(F)$, where $p$ is the covering projection from $\widetilde{M}$ to $M$.

Theorem 5.1. If $M$ is an oriented 3-manifold with $\partial M$ a union of tori, $S$ is a two-sided spun-normal surface dual to a given action of $\pi_{1} M$ on a tree $\mathfrak{T}$, and $T$ is a twisted squares surface produced from the same data, then $T$ is also dual to the action.

Proof. In section 2.4 of [2], Shalen shows how to modify a surface dual to an action by a (disk) compression to produce a new surface which is also dual to the action. He also shows how to remove boundary parallel components. We need to show that the other modification (boundary annulus compression) results in surfaces dual to the action.

If $F$ is a surface dual to an action of $\pi_{1} M$ on $\mathfrak{T}$ with a boundary annulus $A$ as in Figure 11, we can modify $\widetilde{f}$ as follows: Let $N$ be a small tubular neighbourhood

\footnotetext{
${ }^{4}$ In the context of [2] we work with $\pi_{1} M$ acting on $\mathfrak{T}$ without inversions, and by 2.3 .3 of [2] any such dual surfaces must be two-sided.
} 
of $A$ in $M$ (see Figure 13). Lift $F, A$ and $N$ to $\widetilde{F}, \widetilde{A}$ and $\widetilde{N}$ in $\widetilde{M}$. Now let $\widetilde{f}^{\prime}$ be identical to $\widetilde{f}$ outside of $\widetilde{N}$ and be related by a $\pi_{1} M$ equivariant homotopy to $\widetilde{f}$ inside $\widetilde{N}$ which pushes $\widetilde{F}$ out to the boundary, stretching the parts of $\widetilde{N} \backslash \widetilde{F}$ that do not touch $\partial \widetilde{M}$ until they do touch $\partial \widetilde{M}$. The resulting $\widetilde{f}^{\prime}$ is $\pi_{1} M$ equivariant and with a little care can be made to be transverse to $E$. The resulting dual surface $F^{\prime}$ is obtained from $F$ by a boundary annulus compression.

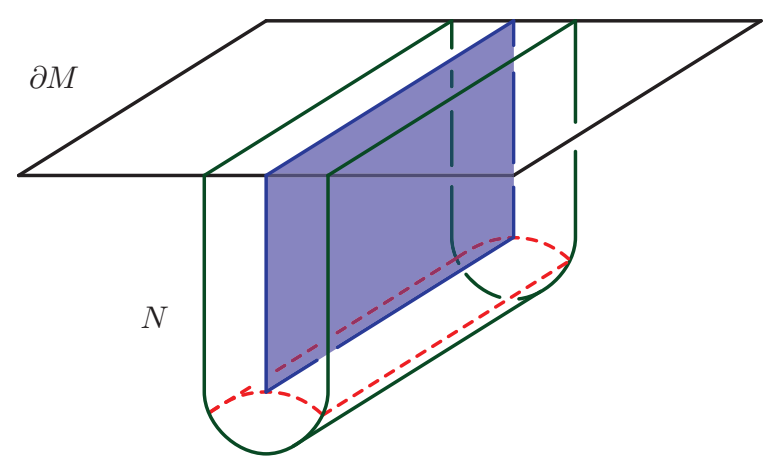

FiguRE 13. A small tubular neighbourhood of a boundary annulus. The part of the surface $F$ outside of $N$ is not shown.

We are now able to prove Theorem 1.2. In the statement of the theorem, "correspondence between an ideal point of the deformation and character varieties" means that as we approach the ideal point in the deformation variety, the corresponding characters approach an ideal point in the character variety. See [3, section 2.5, for more detail on the map between the varieties. We say that a surface is detected by the character variety if it can be produced by the Culler-Shalen construction from an ideal point of the character variety (see [2]). As in Remark 1.3, we do not follow Yoshida [6] in taking double covers of only components that are not two-sided, but rather double up all components or, equivalently, only consider integer solutions that result in two-sided twisted squares surfaces.

Proof of Theorem 1.2. First assume that the spun-normal surface $S$ obtained from the same data as we use to build $T$ is two-sided. Proposition 23 of Tillmann [3] gives us (although not in this language) that the spun-normal surface corresponding to an ideal point of the deformation variety that corresponds to an ideal point of the character variety is dual to the action on a tree associated to the ideal point of the character variety (we need $S$ to be two-sided and hence bicollared for this). The data used to build $T$ from the ideal point is an admissible integer solution to the Q-matching equations, which is the same as for Yoshida's construction, so $T$ and $S$ are related as in Theorem 5.1. Therefore $T$ is also dual to the ideal point of the character variety, and so is any essential surface obtained from $T$ by compressions.

If we are in the case where $T$ is two-sided but $S$ is not, then doubling all of the numbers of quadrilaterals in the construction of the spun-normal surface results in the double cover of $S$ which is two-sided, and so the above argument goes through. The result gives us that the double cover of $T$ (which is what we get by doubling all of the numbers of twisted squares) is dual to the ideal point of the character 
variety. Since $T$ was two-sided already, this surface consists of two parallel components for each component of $T$. We follow the Culler-Shalen construction through with two parallel copies until the very end, at which point we may take only one parallel component, and again we produce the essential surface obtained from $T$ by compressions.

Remark 5.2. It isn't clear if the two constructions (spun-normal and twisted squares surfaces) from an ideal point of the deformation variety can always produce the same essential surfaces, but the above results (and primarily Tillmann's result) do show that in the case that there is a corresponding ideal point of the character variety, all of the essential surfaces we get from either construction can also be obtained by the Culler-Shalen construction. However, explicitly carrying out the algorithms seems to be more feasible for the two deformation variety constructions.

\section{INCOMPRESSIBLE SURFACES IN GENERAL}

We can also draw a connection with incompressible surfaces in general (as opposed to specifically those produced by some degeneration of the geometric structure) using the following theorem:

Theorem 6.1 (Theorem 1.5 of Walsh [5). Let $M$ be an atoroidal, acylindrical, irreducible, compact three-manifold with torus boundary components, and let $\mathcal{T}$ be an ideal triangulation of $M$ with essential edges. Let $S$ be a properly embedded, two-sided, incompressible surface in $M$ that is not a virtual fiber. Then $S$ can be isotoped to be in normal or spunnormal form in $(M, \mathcal{T})$.

Corollary 6.2. With the above notation and conditions, there exists a twisted squares surface $T$ embedded in $M$ such that $T$ can be obtained from $S$ by a number of boundary annulus compressions.

Proof. $S$ is incompressible, so there cannot be any disk compression moves to make, only boundary annulus compressions.

\section{FurTher QUESTIONS}

(1) Are there any manifolds with essential surfaces that can be produced by compressions from only one of the spun-normal and twisted squares constructions and not the other? The difference seems to come down to the boundary annulus compressions.

(2) Are there any manifolds with essential surfaces that can be produced only via the Culler-Shalen construction and not by either the twisted squares or spun-normal constructions? If so, does there exist some other way to construct surfaces using the deformation variety that does obtain all of the essential surfaces that the Culler-Shalen construction does?

(3) Tillmann [3] also asks the reverse question to the above, whether there are closed essential surfaces that are not detected by the character variety but that are detected by the deformation variety (i.e. produced by the spunnormal construction in the case that the ideal point of the deformation variety does not correspond to an ideal point of the character variety). We can expand the question to ask the same for twisted squares surfaces. 
(4) Is there an intrinsic interpretation (not depending on the triangulation of the manifold) of the orientation of curves formed from the boundaries of twisted squares on $\partial M$ in a twisted squares surface? There are parallel curves with opposite orientations if and only if there are boundary annulus compressions to make in altering the spun-normal surface to the twisted squares form, and so understanding these orientations could help us answer the first question in this list.

\section{REFERENCES}

1. Henry Segerman, Detection of incompressible surfaces in hyperbolic punctured torus bundles, arXiv:math/0610302v2.

2. Peter Shalen, Representations of 3-manifold groups, Handbook of Geometric Topology (R.B. Sher and R.J. Daverman, eds.), North-Holland, first ed., 2001. MR.1886685 (2003d:57002)

3. Stephan Tillmann, Degenerations of ideal hyperbolic triangulations, arXiv:math.GT/0508295.

4. _ Normal surfaces in topologically finite 3-manifolds, L'Enseignement Mathématique $\mathbf{5 4}$ (2008), 329-380. MR2478091

5. Genevieve S. Walsh, Incompressible surfaces and spunnormal form, arXiv:math/0503027.

6. Tomoyoshi Yoshida, On ideal points of deformation curves of hyperbolic 3-manifolds with one cusp, Topology 30 (1991), no. 2, 155-170. MR1098911 (92a:57018)

Department of Mathematics, The University of Texas at Austin, 1 University Station C1200, Austin, Texas 78712-0257

E-mail address: henrys@math.utexas.edu 